\title{
Nanofluid flow and heat transfer in a microchannel with
}

\section{interfacial electrokinetic effects}

\author{
Qingkai Zhao ${ }^{1, a, b} \quad$ Hang $\mathrm{Xu}^{1, b}, \quad$ Longbin $\mathrm{Tao}^{c}$ \\ ${ }^{a}$ School of Shipbuilding Engineering, Harbin Engineering University, Harbin 150001, China \\ ${ }^{b}$ Collaborative Innovation Center for Advanced Ship and Deep-Sea Exploration (CISSE), \\ State Key Lab of Ocean Engineering, School of Naval Architecture, Ocean and Civil \\ Engineering, Shanghai Jiao Tong University, Shanghai 200240, China \\ ${ }^{c}$ Department of Naval Architecture, Ocean and Marine Engineering, University of \\ Strathclyde, Glasgow G4 0LZ, United Kingdom
}

\begin{abstract}
The behaviour of microchannel flow of a nanofluid between two parallel flat plates in the presence of the electrical double layer (EDL) is investigated in this paper. The problem is formulated based on the Buongiorno nanofluid model with the electrical body force due to the EDL being considered in the momentum equation. As one of the highlights of the present investigation, the unphysical assumption introduced in previous studies often leading to the discontinuities of flow field that the electrostatic potential in the middle of the channel has to be equal to zero is eliminated. In addition, the inappropriate assumption that the pressure constant is treated as a known condition is also rectified. The new model is developed with the governing equations being reduced by a set of dimensionless quantities to a set of coupled ordinary differential equations. Based on the analytical approximations, the influences of various physical parameters on the flow field and temperature field, and the important physical quantities of practical interests are analysed and discussed in detail.

1 These authors contributed equally.

Email:qkzhao86@163.com (Q.K. Zhao), hangxu@sjtu.edu.cn (H. Xu)

The corresponding author: longbin.tao@strath.ac.uk (L. Tao)
\end{abstract}


Keywords electrical double layer, microchannel, nanofluid, zeta potential, analytical solution

\section{Nomenclature}

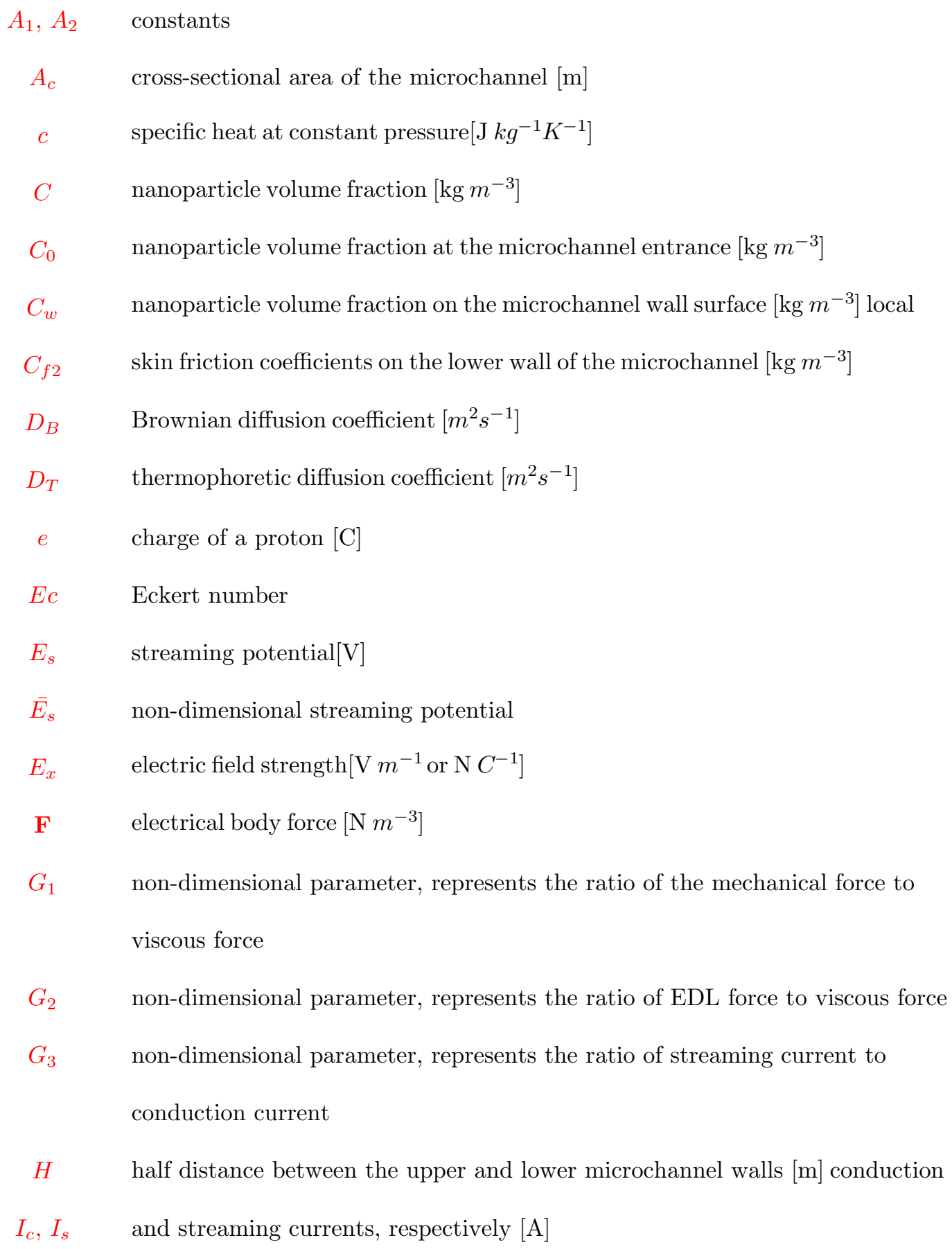




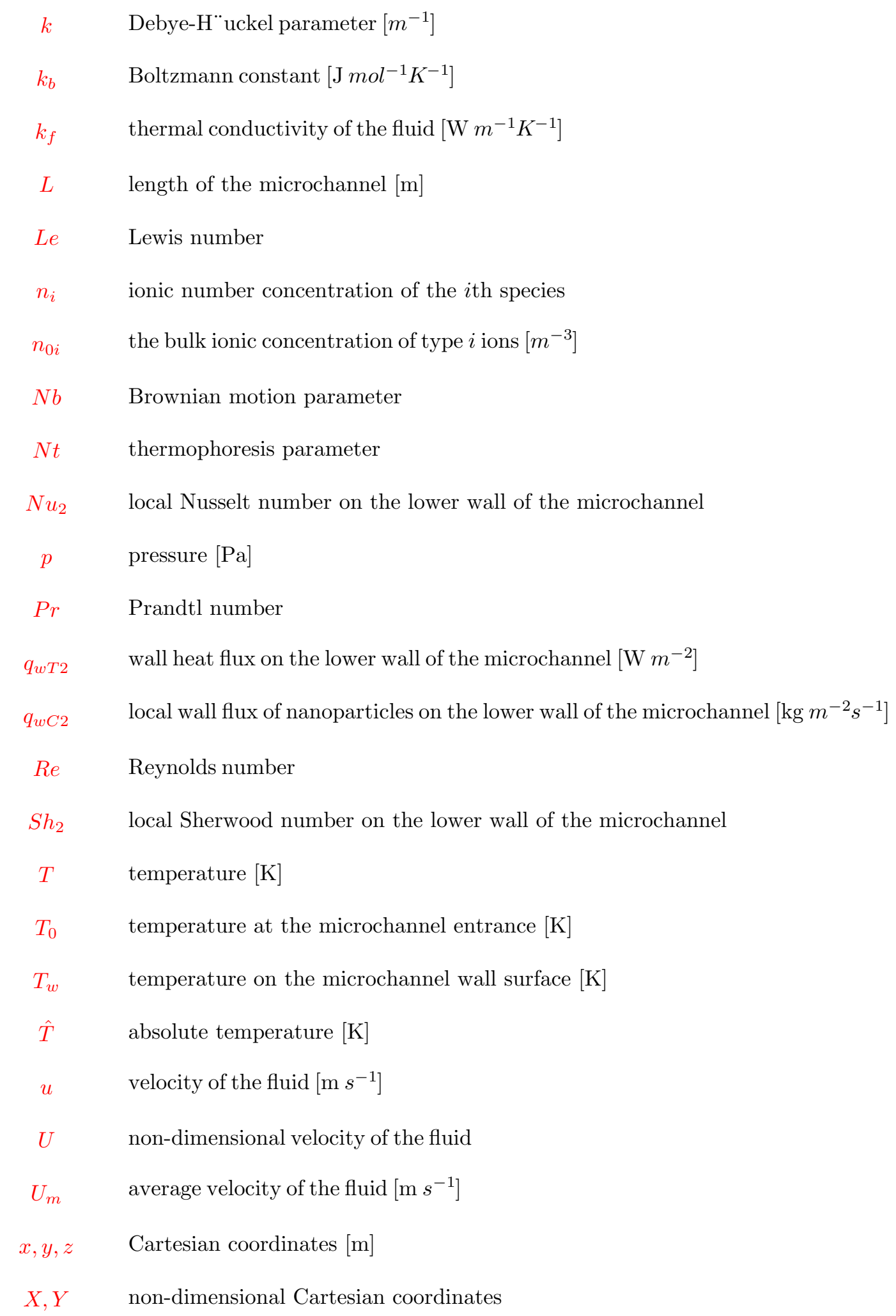


$\hat{z}_{i} \quad$ the valence of type $i$ ions

\section{Greek symbols}

\begin{tabular}{|c|c|}
\hline$\alpha$ & thermal diffusivity of the nanofluid $\left[\mathrm{m}^{2} \mathrm{~s}^{-1}\right]$ \\
\hline$\varepsilon$ & dielectric constant of the medium \\
\hline$\varepsilon_{0}$ & permittivity of vacuum $\left[\mathrm{C} V^{-1} m^{-1}\right]$ \\
\hline$\kappa$ & non-dimensional electrokinetic separation distance between the upper \\
\hline & and lower wall of the microchannel \\
\hline$\lambda_{0}$ & electrical conductivity of the fluid $\left[\Omega^{-1} m^{-1}\right]$ \\
\hline$\mu$ & dynamic viscosity of the fluid $\left[\mathrm{kg} m^{-1} s^{-1}\right]$ \\
\hline$\nu$ & kinematic viscosity of the fluid $\left[m^{2} s^{-1}\right]$ \\
\hline$\Theta$ & non-dimensional temperature distribution \\
\hline$\Phi$ & non-dimensional nanoparticle volume fraction \\
\hline$\rho_{e}$ & charge density $\left[\mathrm{C}^{-3}\right]$ \\
\hline$\rho_{f}$ & density of the fluid $[\mathrm{kg} \mathrm{m}-3]$ \\
\hline$\tau$ & ratio of the heat capacity of the nanoparticle to that of the fluid shear \\
\hline$\tau_{w 2}$ & stress on the lower wall of the microchannel $[\mathrm{Pa}]$ \\
\hline$\zeta$ & zeta potential $[\mathrm{V}]$ \\
\hline$\psi$ & electrostatic potential $[\mathrm{V}]$ \\
\hline 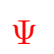 & non-dimensional electrostatic potential \\
\hline
\end{tabular}

\section{Introduction}

The research of fluid flow and heat transfer in microchannel is of significant interest to engineers and scientists in industrial applications such as microchannel heat sinks for cooling high power very large scale integration circuitry and laser diode arrays, heat transfer 
augmentation in aerospace technology, micro-reactors for the analysis of biological cells and micro fluid pumps $[1,2]$. However, conventional transport theories are insufficient to explain many phenomena associated with microscale flow. Experimental observations [3-5] have shown that flow and heat transfer behaviours in microscale are quite different from those in macroscale. Particularly, Wang and Peng [5] noticed that transition and laminar heat transfer in microchannels are highly strange and complicated compared with the conventionally sized situation. They conjectured that this unusual behavior of microchannel flow may be largely due to electrical double layer (EDL) effects. If the liquid contains very small number of ions, the electrostatic charges on the solid surface will attract the counterions in the liquid to establish an electrical field. The rearrangement of the electrostatic charges on the solid surface and the balancing charges in the liquid is called the EDL [6]. When a liquid is forced through a microchannel under hydrostatic pressure, the ions in the diffuse layer of the EDL are carried towards the downstream end. This causes an electrical current, called streaming current. The accumulation of ions downstream sets up an electrical field with an electrical potential called the streaming potential. This field causes a current, called conduction current, to flow back in the opposite direction. When conduction current is equal to the streaming current a steady state is reached. It is easy to understand that, when the ions are moved in the diffuse double layer, they pull the liquid along with them. However, the motion of the ions in the diffuse double layer is subject to the electrical potential of the double layer. Thus the liquid flow and associated heat transfer are affected by the presence of the EDL.

Generally, for macrochannel flow the EDL effects can be neglected since the EDL thickness is very small as compared to the channels' characteristic length. While for microchannel flow, the thickness of the EDL is comparable to the characteristic length of channels and its effect has to be considered. It is noted that the EDL effects originated from the interfacial 
electrokinetic effects [7] by the variation of electric potential near a surface and could have a significant influence on the behaviour of fluid flow. Therefore, it is necessary to investigate the fundamental characteristics of these phenomena in order to develop the high quality products. Recently, Mala et al. [8] analyzed the effects of the EDL at the solid-liquid interface on liquid flow and heat transfer through a microchannel between two parallel plates. Mala et al. [9] reported experimental results on flow of distilled water and aqueous solutions through silicon and glass microchannels between two parallel plates. Ren et al. [10] further investigated the electro-viscous effect caused by the EDL near a solid-liquid interface in microchannels. Zhang et al. [11] experimentally studied the streaming potentials across a porous membrane in various organic-aqueous solutions.

On the other hand, increasing research effort has been devoted to study the mechanism of nanofluids owing to their great potentials in thermal engineering [12-14]. Many experiments were carried out to investigate convective flow and heat transfer features of various nanofluids [15-17]. Among those studies, Wen and Ding [15] experimentally confirmed that the heat transfer enhancement is prominent when pure heat transfer fluids are replaced by nanofluids. Similar conclusions were drawn by other researchers $[18,19]$. Theoretically, several mathematical models such as the homogenous flow model [20], the dispersion model [21], the Buongiorno's model [22] have been suggested to predict nanofluids' behaviours. Among these models, the Buongiorno's model received great attention [23] since it explains well the slip mechanisms between the nanoparticles and the base fluid. Since the volumetric distributions of nanoparticles can be altered by virous physical processing, such as fluid flow, heat transfer, electric field, it is very attractive to investigate such multiple physical phenomena with consideration of how these physical processing interacts each other. For example, the EDL modifies the fluid motion obviously, which could affect heat transfer, and also play an important role in the volumetric distribution of the nanoparticles. Therefore, 
it is necessary to investigate the influence of EDL on liquid flow of nanofluids.

This paper is to examine a steady-state, fully-developed, laminar nanofluid flow in a horizontal microchannel with the interfacial electrokinetic effects. The electrical body force resulting from the electrical double layer (EDL) and the electrokinetic fields are considered in the momentum equation. The energy and the volumetric concentration of the nanoparticles equations are established based on the Buongiorno's model. One nonphysical assumption by Mala et al. $[8,9]$ that the electrostatic potential in the middle of the channel has to be equal to zero is corrected since it can lead to the discontinuities of the flow field. The other inappropriate assumption by Mala et al. $[8,9]$ and Ren et al. $[10]$ that the pressure constant is a known condition is also rectified, which ensure our model to agree with commonly-accepted models in the field of fluid mechanics. The governing equation is reduced by non-dimensional variables to a set of coupled nonlinear ordinary equations. Particularly, an analytical solution for the electrical field is presented. Analytical approximations for other fields are obtained by the homotopy analysis næthod. The influences of various physical parameters on important physical quantities of practical interests are analysed and discussed. The studies of these fundamental phenomena and their næchanisms are helpful for the optimal design, inproved performances and broad applications of micro/nanofluidic systems.

\section{Analytical solution to the electrostatic potential}

According to the theory of electrostatics, the relation between $\psi$ and $\rho_{e}$ is given by the Poisson's equation near a flat surface as $[6,24]$

$$
\frac{d^{2} \psi}{d y^{2}}=-\frac{\rho_{e}}{\varepsilon_{0} \varepsilon},
$$

where $\varepsilon$ is the dielectric constant of the fluid and $\varepsilon_{0}$ is the permittivity of vacuum. 
Using the assumption of the equilibrium Boltzmann distribution about uniform dielectric constant and neglecting fluctuation, the number of ion distribution in a symmetric electrolyte solution takes the form

$$
n_{i}=n_{0 i} \exp \left(-\frac{\hat{z}_{i} e \psi}{k_{b} \hat{T}}\right),
$$

where $n_{0 i}$ and $\hat{z}_{i}$ denote the bulk ionic concentration and the valence of type $i$ ions, respectively, $e$ the charge of a proton, $\psi$ the electrical potential, $k_{b}$ the Boltzmann's constant and $\hat{T}$ the absolute temperature. The net charge density in a unit volume of the fluid is given by

$$
\rho_{e}=\left(n_{+}-n_{-}\right) \hat{z} e=-2 n_{0} \hat{z} e \sinh \left(\frac{\hat{z} e \psi}{k_{b} \hat{T}}\right)
$$

Substituting Eq.(3) into the Poisson equation (1), we obtain the well-known Poisson-Boltzmann equation

$$
\frac{d^{2} \psi}{d y^{2}}=\frac{2 n_{0} \hat{z} e}{\varepsilon_{0} \varepsilon} \sinh \left(\frac{\hat{z} e \psi}{k_{b} \hat{T}}\right) .
$$

Eq.(4) can be non-dimensionalized, via the similarity variables

$$
X=\frac{x}{H}, \quad Y=\frac{y}{H}, \quad \Psi(Y)=\frac{\hat{z} e \psi}{k_{b} \hat{T}}, \quad \rho^{*}(Y)=\frac{\rho_{e}}{n_{0} \hat{z} e},
$$

in the following forms

$$
\begin{aligned}
& \frac{d^{2} \Psi(Y)}{d Y^{2}}=\kappa^{2} \sinh (\Psi(Y)) . \\
& \frac{d^{2} \Psi(Y)}{d Y^{2}}=-\frac{\kappa^{2}}{2} \rho^{*}(Y) .
\end{aligned}
$$

where $\kappa=H k$ in which $k^{2}=2 n_{0} \hat{z}^{2} e^{2} /\left(\varepsilon_{0} \varepsilon k_{b} \hat{T}\right)$ is the Debye-Hückel parameter, and $1 / k$ is normally regard as the EDL thickness.

The appropriate boundary conditions for Eqs.(6) and (7) are

$$
\Psi( \pm 1)=\bar{\zeta}=\frac{\hat{z} e \zeta}{k_{b} \hat{T}}
$$


where $\zeta$ is the zeta potential measuring the electrical potential at the shear plane, i.e., the boundary between the compact layer and the diffuse layer [6].

If the electrical potential is small compared to the thermal energy of the ions, i.e., $\left(|\hat{z} e \psi|<\left|k_{b} \hat{T}\right|\right)$, using the Debye-Hückel linear approximation, Eq.(6) takes the form

$$
\frac{d^{2} \Psi(Y)}{d Y^{2}}=\kappa^{2} \Psi(Y)
$$

which has the analytical solution

$$
\Psi(Y)=\frac{\bar{\zeta}}{1+e^{2 \kappa}}\left[e^{\kappa(1+Y)}+e^{\kappa(1-Y)}\right]
$$

\section{Mathematical formulation for other fields}

The nanofluid flow and heat transfer through a horizontal rectangular microchannel in the presence of the effects of EDL is investigated. The physical sketch is shown in Fig.1. Here $x$-axis is paralleled to the channel walls and $y$-axis is perpendicular to the walls. The origin of the coordinates is fixed at the centerline of the microchannel. $H$ is the half distance between the upper and lower walls, $L$ is the length of the microchannel, $W$ is the width of the microchannel. To simplify the model without violating real physical circumstances, we assume that $W \gg H$, so that the problem can be formulated as a two-dimensional nonlinear microchannel flow problems in the presence of EDL effects.

The governing equations including the conservations of the total mass, the momentum, 
the thermal energy and the nanoparticle volumetric fraction are expressed by

$$
\begin{aligned}
& \nabla \cdot \mathbf{V}=0, \\
& \rho(\mathbf{V} \cdot \nabla) \mathbf{V}=-\nabla p+\mu \nabla^{2} \mathbf{V}+\mathbf{F}, \\
& (\mathbf{V} \cdot \nabla) T=\alpha \nabla^{2} T+\tau\left[D_{B} \nabla T \cdot \nabla C+\left(\frac{D_{T}}{T_{0}}\right) \nabla T \cdot \nabla T\right]+\frac{\mu}{\rho c} \Phi, \\
& (\mathbf{V} \cdot \nabla) C=D_{B} \nabla^{2} C+\left(\frac{D_{T}}{T_{0}}\right) \nabla^{2} T,
\end{aligned}
$$

where $\mathbf{F}$ is the electrical body force. $\Phi$ is the viscous dissipation term, defined by

$$
\begin{aligned}
& \Phi=2\left[\left(\frac{\partial u}{\partial x}\right)^{2}+\left(\frac{\partial v}{\partial y}\right)^{2}+\left(\frac{\partial w}{\partial z}\right)^{2}\right]+\left(\frac{\partial v}{\partial x}+\frac{\partial u}{\partial y}\right)^{2}+\left(\frac{\partial w}{\partial y}+\frac{\partial v}{\partial z}\right)^{2} \\
& +\left(\frac{\partial u}{\partial z}+\frac{\partial w}{\partial x}\right)^{2}-\frac{2}{3}\left(\frac{\partial u}{\partial x}+\frac{\partial v}{\partial y}+\frac{\partial w}{\partial z}\right)^{2} .
\end{aligned}
$$

For parallel flow in channels, it is known that only one velocity component is not equal to zero, that means all fluid particles moving in the same direction. If, for example, only the velocity component $u$ is nonzero, and thus $v$ is everywhere zero, it follows immediately from the continuity equation that $\partial u / \partial x=0$ and therefore $u$ is independent of $x$. Similarly, the hydraulic pressure $p$ is only dependent on the fluid motion, which indicates that it is only a function of $x$ and therefore the pressure gradient $d p / d x$ is constant. It is assumed that the temperature and the nanoparticle volumetric fraction on both walls increase or decrease linearly with $x$, namely, $T_{w}(x)=T_{0}+A_{1} x$ and $C_{w}(x)=C_{0}+A_{2} x$, where $T_{0}$ and $C_{0}$ are the reference temperature and the reference nanoparticle volumetric fraction at the channel entrance, respectively. Since the temperature $T$ and the nanoparticle volumetric fraction $C$ vary linearly with $x[25,26]$, we obtain $\frac{\partial^{2} T}{\partial x^{2}}=\frac{\partial^{2} C}{\partial x^{2}}=0$.

Under those assumptions, the continuity equation is automatically satisfied, and other 
governing equations are reduced to

$$
\begin{aligned}
\mu \frac{\partial^{2} u}{\partial y^{2}} & -\frac{\partial p}{\partial x}+E_{x} \rho_{e}=0 \\
u \frac{\partial T}{\partial x} & =\alpha \frac{\partial^{2} T}{\partial y^{2}}+\tau D_{B}\left(\frac{\partial T}{\partial x} \frac{\partial C}{\partial x}+\frac{\partial T}{\partial y} \frac{\partial C}{\partial y}\right) \\
& +\frac{\tau D_{T}}{T_{0}}\left[\left(\frac{\partial T}{\partial x}\right)^{2}+\left(\frac{\partial T}{\partial y}\right)^{2}\right]+\frac{\mu}{\rho c}\left(\frac{\partial u}{\partial y}\right)^{2}, \\
u \frac{\partial C}{\partial x} & =D_{B} \frac{\partial^{2} C}{\partial y^{2}}+\frac{D_{T}}{T_{0}} \frac{\partial^{2} T}{\partial y^{2}},
\end{aligned}
$$

subject to the boundary conditions

$$
u( \pm H)=0, \quad T_{w}( \pm H)=T_{0}+A_{1} x, \quad C_{w}( \pm H)=C_{0}+A_{2} x
$$

It is a common practice in channel flow studies to assume the mass flow rate as a prescribed quantity. We thus obtain

$$
U_{m}=\frac{1}{2 H} \int_{-H}^{+H} u(y) d y=\frac{1}{H} \int_{0}^{+H} u(y) d y
$$

where $U_{m}$ is the average fluid velocity in the channel section.

Define the dimensionless quantities

$$
U(Y)=\frac{u}{U_{m}}, \quad \Theta(Y)=\frac{T-T_{w}}{A_{1} H}, \quad \Phi(Y)=\frac{C-C_{w}}{A_{2} H} .
$$

Non-dimensionalize the momentum equation (16) by similarity transformations (5) and (21), we obtain

$$
\frac{d^{2} U(Y)}{d Y^{2}}+G_{1}-2 G_{2} \bar{E}_{s} \Psi(Y)=0
$$

subjected to the boundary conditions

$$
U( \pm 1)=0, \quad \int_{0}^{1} U(Y) d Y=1
$$

where

$$
G_{1}=\frac{H^{2}}{\mu U_{m}} P_{x}, \quad G_{2}=\frac{n_{0} \hat{z} e \zeta H^{2}}{\mu U_{m} L},
$$


in which $P_{x}=-\frac{d p}{d x}$ is the pressure constant, $\bar{E}_{s}=E_{s} / \zeta$ is the stream potential, and $E_{x}=E_{s} / L$

Substituting the non-dimensional variables (21) into Eqs.(17) and (18), we obtained the reduced energy equation and concentration of nanoparticles equation as

$$
\begin{aligned}
& \Theta^{\prime \prime}+N b\left(1+\Theta^{\prime} \Phi^{\prime}\right)+N t\left(1+\Theta^{\prime 2}\right)+\operatorname{Pr} E c U^{\prime 2}-\operatorname{Re} \operatorname{Pr} U=0, \\
& \Phi^{\prime \prime}+\frac{N t}{N b} \Theta^{\prime \prime}-\operatorname{Re} \operatorname{Pr} L e U=0,
\end{aligned}
$$

subject to following boundary conditions

$$
\Theta( \pm 1)=0, \quad \Phi( \pm 1)=0
$$

where $N b=\frac{\tau D_{B} A_{2} H}{\alpha}$ is the Brownian motion parameter, $N t=\frac{\tau D_{T} A_{1} H}{\alpha T_{0}}$ is the thermophoresis parameter, $\operatorname{Pr}=\frac{\nu}{\alpha}$ is the Prandtl Number, $R e=\frac{U_{m} H}{\nu}$ is the Reynolds Number, $E c=\frac{U_{m}^{2}}{c A_{1} H}$ is the Eckert number, $L e=\frac{\alpha}{D_{B}}$ is the Lewis number, respectively.

The physically important quantities of practical interests are the local skin friction, the local Nusselt number, the local Sherwood number. Since the flow is symmetric in the channel, we only need to consider them on the lower wall. In this case, they are defined by

$$
C_{f 2}=\frac{\tau_{w 2}}{\rho_{f} U_{m}^{2}}, N u_{2}=\frac{x q_{w T 2}}{k_{f}\left(T_{w}-T_{0}\right)}, \quad S h_{2}=\frac{x q_{w C 2}}{D_{B}\left(C_{w}-C_{0}\right)}
$$

where

$$
\tau_{w 2}=\mu\left(\frac{\partial u}{\partial y}\right)_{y=-H}, \quad q_{w T 2}=-k_{f}\left(\frac{\partial T}{\partial y}\right)_{y=-H}, \quad q_{w C 2}=-D_{B}\left(\frac{\partial C}{\partial y}\right)_{y=-H} .
$$

Substituting Eq.(21) into Eq.(27), we obtain

$$
C_{f 2}=\frac{1}{R e} U^{\prime}(-1), \quad N u_{2}=-\Theta^{\prime}(-1), \quad S h_{2}=-\Phi^{\prime}(-1) .
$$




\section{Explicit solution for the flow field}

From Eq.(22) and its boundary conditions (23), the analytical solution for the reduced velocity field is obtained as

$$
U(Y)=\frac{G_{1}}{2}\left(1-Y^{2}\right)+\frac{2 \bar{E}_{s} G_{2} \bar{\zeta}}{\kappa^{2}}\left[\frac{\cosh (\kappa Y)}{\cosh (\kappa)}-1\right]
$$

It is noted that the pressure constant $G_{1}$ and the dimensionless streaming potential $\bar{E}_{s}$ are not known yet, which will be determined as follow. Using the integral boundary conditions for $U(Y)$ denoted in Eq.(23), the relationship between $G_{1}$ and $\bar{E}_{s}$ are obtained:

$$
\frac{G_{1}}{3}-\frac{2 \bar{E}_{s} G_{2} \bar{\zeta}}{\kappa^{2}}+\frac{2 \bar{E}_{s} G_{2} \bar{\zeta} \sinh (\kappa)}{\kappa^{3} \cosh (\kappa)}=1 .
$$

Physically, it is known that the electrical field is generated as an electrolyte is driven by a hydrostatic pressure through a microchannel with charged walls, which results in a streaming current, defined by

$$
I_{s}=\int_{A_{c}} u \rho_{e} d A_{c}
$$

Using Eqs.(5), (7), and (21), we obtain its dimensionless form

$$
I_{s}=-4 U_{m} n_{0} \hat{z} e W H \int_{0}^{1} U(Y) \Psi(Y) d Y .
$$

On the other hand, the streaming potential produces a conduction current in the reverse direction, which is given by

$$
I_{c}=\frac{\lambda_{0} E_{s} A_{c}}{L}
$$

where $\lambda_{0}$ is the constant electrical conductivity of the fluid, $A_{c}$ is the cross-sectional area of the channel. It can be transformed, using the relations $\bar{E}_{s}=E_{s} / \zeta$ and $A_{c}=2 H \times W$, into the following form

$$
I_{c}=\lambda_{0} \zeta \frac{2 H W}{L} \bar{E}_{s}
$$


The electrokinetic potential $E_{x}$ can be obtained by balancing the streaming current and the electrical conduction current at the steady state. In this situation, the net electrical current equates to zero, which indicates

$$
I=I_{s}+I_{c}=0 .
$$

Using Eqs.(34) and (32), $\bar{E}_{s}$ is obtained

$$
\bar{E}_{s}=2 G_{3} \int_{0}^{1} U(Y) \Psi(Y) d Y,
$$

where $G_{3}=\frac{L U_{m} n_{0} \hat{z} e}{\lambda_{0} \zeta}$ is a constant.

Substituting Eqs.(10) and (30) into Eq.(36), the other relationship between $G_{1}$ and $\bar{E}_{s}$ is obtained:

$$
\bar{E}_{s}-\frac{2 G_{3} \bar{\zeta}\left[G_{1} \kappa+\bar{E}_{s} G_{2} \kappa \bar{\zeta} \operatorname{sech}^{2}(\kappa)-\left(G_{1}+\bar{E}_{s} G_{2} \bar{\zeta}\right) \tanh (\kappa)\right]}{\kappa^{3}}=0 .
$$

Therefore, $G_{1}$ and $\bar{E}_{s}$ can be determined, via combining Eq.(31) and Eq.(37), as

$$
\begin{aligned}
& G_{1}=\frac{3 \kappa^{6}-6 \kappa^{3} G_{2} G_{3} \bar{\zeta}^{2}\left[\kappa \operatorname{Sech}^{2}(\kappa)-\tanh (\kappa)\right]}{\kappa^{6}-2 G_{2} G_{3} \bar{\zeta}^{2}\left[6 \kappa^{2}+\kappa^{4} \operatorname{Sech}^{2}(\kappa)-\kappa\left(12+\kappa^{2}\right) \tanh (\kappa)+6 \tanh ^{2}(\kappa)\right]}, \\
& \bar{E}_{s}=\frac{6 G_{3} \kappa^{3} \bar{\zeta}[\kappa-\tanh (\kappa)]}{\kappa^{6}-2 G_{2} G_{3} \bar{\zeta}^{2}\left[6 \kappa^{2}+\kappa^{4} \operatorname{sech}^{2}(\kappa)-\kappa\left(12+\kappa^{2}\right) \tanh (\kappa)+6 \tanh ^{2}(\kappa)\right]} .
\end{aligned}
$$

Hence the explicit solution $U(Y)$ denoted in Eq.(30) is fully determined.

\section{HAM solution and discussion}

It is worth mentioning that the Eq.(22) contains two unknown constants $G_{1}$ and $\int_{0}^{1} U(Y) \Psi(Y) d Y$, which are difficult to be calculated directly by either numerical or analytical methods without special treatments. To overcome this limitation, we employ and extend the homotopy analysis method (HAM) [27] to obtain the accurate solutions of the nonlinear equations Eqs.(9), (22), (24) and (25), in which the exact values for $G_{1}$ and $\int_{0}^{1} U(Y) \Psi(Y) d Y$, are 
calculated spontaneously and simultaneously as part of the solution procedure without any approximations.

We first define $G_{1}$ and $\int_{0}^{1} U(Y) \Psi(Y) d Y$ as

$$
G_{1}=\sigma=\sigma_{0}+\sum_{j=1}^{\infty} \sigma_{j}, \quad \int_{0}^{1} U \Psi d Y=w=w_{0}+\sum_{j=1}^{\infty} w_{j}
$$

then express the functions $\Psi(Y), U(Y), \Theta(Y)$ and $\Phi(Y)$ as

$$
\begin{gathered}
\Psi(Y)=\psi_{0}(Y)+\sum_{j=1}^{\infty} \psi_{j}(Y), \quad U(Y)=u_{0}(Y)+\sum_{j=1}^{\infty} u_{j}(Y), \\
\Theta(Y)=\theta_{0}(Y)+\sum_{j=1}^{\infty} \theta_{j}(Y), \quad \Phi(Y)=\phi_{0}(Y)+\sum_{j=1}^{\infty} \phi_{j}(Y) .
\end{gathered}
$$

In the framework of the HAM, the $k$ th order HAM deformation equations can be written as

$$
\begin{aligned}
\psi_{m}^{\prime \prime}-\chi_{m} \psi_{m-1}^{\prime \prime} & =\hbar_{\psi} R_{\psi, m}, \\
u_{m}^{\prime \prime}-\chi_{m} u_{m-1}^{\prime \prime} & =\hbar_{u} R_{u, m}, \\
\theta_{m}^{\prime \prime}-\chi_{m} \theta_{m-1}^{\prime \prime} & =\hbar_{\theta} R_{\theta, m}, \\
\phi_{m}^{\prime \prime}-\chi_{m} \phi_{m-1}^{\prime \prime} & =\hbar_{\phi} R_{\phi, m} .
\end{aligned}
$$

subject to the boundary conditions

$$
\psi_{m}( \pm 1)=0, \quad u_{m}( \pm 1)=0, \quad \int_{0}^{1} u_{m}=1, \quad \theta_{m}( \pm 1)=0, \quad \phi_{m}( \pm 1)=0,
$$

where $\hbar_{\psi}, \hbar_{u}, \hbar_{\theta}$ and $\hbar_{\phi}$ are the HAM auxiliary parameters used for the convergence-control of the HAM analytical approximations. Also, $R_{\psi, m}, R_{u, m}, R_{\theta, m}, R_{\phi, m}$ and $\chi_{m}$ are defined, 
respectively, by

$$
\begin{aligned}
R_{\psi, m}= & \psi_{m-1}^{\prime \prime}-\kappa^{2} \psi_{m-1} \\
R_{u, m}= & u_{m-1}^{\prime \prime}+\sigma_{m-1}-4 G_{2} G_{3} \sum_{j=0}^{m-1} w_{j} \psi_{m-1-j}, \\
R_{\theta, m}= & \theta_{m-1}^{\prime \prime}+\sum_{j=0}^{m-1}\left(N b \theta_{j}^{\prime} \phi_{m-1-j}^{\prime}+N t \theta_{j}^{\prime} \theta_{m-1-j}^{\prime}+\operatorname{PrEc} u_{j}^{\prime} u_{m-1-j}^{\prime}\right) \\
& -\operatorname{Re} \operatorname{Pr} u_{m-1}+(N t+N b) *\left(1-\chi_{m}\right) \\
R_{\phi, m}= & \phi_{m-1}^{\prime \prime}+\frac{N t}{N b} \theta_{m-1}^{\prime \prime}-\operatorname{Re} \operatorname{Pr} \operatorname{Le} u_{m-1},
\end{aligned}
$$

where

$$
\chi_{m}= \begin{cases}0 & m \leq 1, \\ 1 & m>1 .\end{cases}
$$

Here the expression (36) is used to obtain $R_{u, m}$.

The solutions of Eqs.(40)-(43) are in the forms of

$$
\begin{aligned}
& \psi_{m}=\psi^{*}+\chi_{m} \psi_{m-1}+C_{1, m}+C_{2, m} Y, \\
& u_{m}=u^{*}+\chi_{m} u_{m-1}+C_{3, m}+C_{4, m} Y, \\
& \theta_{m}=\theta^{*}+\chi_{m} \theta_{m-1}+C_{5, m}+C_{6, m} Y, \\
& \phi_{m}=\phi^{*}+\chi_{m} \phi_{m-1}+C_{7, m}+C_{8, m} Y,
\end{aligned}
$$

where

$$
\begin{aligned}
\psi^{*} & =\iint \hbar_{\psi} R_{\psi, m} d Y d Y, \quad u^{*}=\iint \hbar_{u} R_{u, m} d Y d Y, \\
\theta^{*} & =\iint \hbar_{\theta} R_{\theta, m} d Y d Y, \quad \phi^{*}=\iint \hbar_{\phi} R_{\phi, m} d Y d Y .
\end{aligned}
$$

The integral constants $C_{i, m}(i=1,2,3,4,5,6,7,8)$ are determined by the boundary conditions

$$
C_{1, m}=-\frac{\psi^{*}(1)+\psi^{*}(-1)}{2}, \quad C_{2, m}=-\frac{\psi^{*}(1)-\psi^{*}(-1)}{2}, \quad C_{3, m}=-\frac{u^{*}(1)+u^{*}(-1)}{2}
$$




$$
\begin{gathered}
C_{4, m}=-\frac{u^{*}(1)-u^{*}(-1)}{2}, \quad C_{5, m}=-\frac{\theta^{*}(1)+\theta^{*}(-1)}{2}, C_{6, m}=-\frac{\theta^{*}(1)-\theta^{*}(-1)}{2} \\
C_{7, m}=-\frac{\phi^{*}(1)+\phi^{*}(-1)}{2}, \quad C_{8, m}=-\frac{\phi^{*}(1)-\phi^{*}(-1)}{2} .
\end{gathered}
$$

The above HAM solution procedure can work accordingly after the initial approximations $\psi_{0}(Y), u_{0}(Y), \theta_{0}(Y)$ and $\phi_{0}(Y)$ are chosen properly based on the boundary conditions (44), such as

$$
\begin{aligned}
& \psi_{0}(Y)=\bar{\zeta} Y^{2}, \quad u_{0}(Y)=1+\frac{3}{2} Y^{2}-\frac{5}{2} Y^{4} \\
& \theta_{0}(Y)=1-Y^{2}, \quad \phi_{0}(Y)=1-Y^{2}
\end{aligned}
$$

During the HAM solution procedure, the unknown constant $G_{1}$ is determined in the following way. It is known that $u_{m}$ contains the unknown term $\sigma_{m-1}$, which is determined using the mass flow rate equation(20). For instance, in the case of $\hbar_{u}=-1, G_{2}=G_{3}=1, \bar{\zeta}=1$, for the zero-th order approximation when $m=1$, it is easily seen that $u_{1}$ contains the unknown term $\sigma_{0}$. By means of Eq.(20) we have

$$
\int_{0}^{1} u_{1} d Y=0
$$

we can obtained the value of $\sigma_{0}=\frac{1691}{525}$ from above equation. Also, the constant integration term $w_{0}=\int_{0}^{1} u_{0}(Y) \psi_{0}(Y) d Y$ can be obtained by means of Eq.(55). In this way, the whole solution series in Eq.(38) for $G_{1}$ and the constant integration term can be determined successively from $m=1,2,3, \cdots$.

To check the accuracies of the results, the error estimation function is defined as

$$
E(m)=\max \left\{E_{\Psi}(m), E_{U}(m), E_{\Theta}(m), E_{\Phi}(m)\right\},
$$


where

$$
\begin{aligned}
& E_{\Psi}(m)=\int_{-1}^{1}\left(\Psi^{\prime \prime}-\kappa^{2} \Psi\right)^{2} d Y, \\
& E_{U}(m)=\int_{-1}^{1}\left[U^{\prime \prime}+G_{1}-\left(4 G_{2} G_{3} \int_{0}^{1} U \Psi d Y\right) \Psi\right]^{2} d Y, \\
& E_{\Theta}(m)=\int_{-1}^{1}\left[\Theta^{\prime \prime}+N b\left(1+\Theta^{\prime} \Phi^{\prime}\right)+N t\left(1+\Theta^{\prime 2}\right)+\operatorname{PrEc} U^{\prime 2}-\operatorname{RePr} U\right]^{2} d Y, \\
& E_{\Phi}(m)=\int_{-1}^{1}\left(\Phi^{\prime \prime}+\frac{N t}{N b} \Theta^{\prime \prime}-\operatorname{RePr} \operatorname{Pe} U\right)^{2} d Y .
\end{aligned}
$$

Substituting $m$ th order computational results into Eq.(57), the corresponding error can be obtained. In present work, the study mainly f ocuses on the effect of various physical quantities with the $\kappa$ value ranging from 0 to 20 . The rest of the dimensionless parameters are given the fixed values. For instance, in the case of $G_{2}=G_{3}=1, E c=L e=R e=$ $1, \bar{\zeta}=1, \operatorname{Pr}=5, N t=1 / 10, N b=2 / 10$, we obtain the maximum error $E(m)$ for various values of $\kappa$, as listed in Table 2. Note that the Homotopy-Padé technique [27] is employed to improve the convergence of the HAM approximations.

It is noted that Mala et al. [8] gave an analytical solution for $\Psi(y)$, by imposing an additional boundary condition $\Psi(0)=0$, as

$$
\Psi(Y)=\frac{\bar{\zeta}}{\sinh (\kappa)}|\sinh (\kappa Y)| .
$$

The solution exists singularity at $Y=0$ and is physically unrealistic, as shown in Fig.2. It can be seen in the figure that the present analytical solution denoted in Eq.(10) are structurally different from the result given by Mala et al. $[8,9]$. They are smooth for all $\kappa$. As $\kappa$ becomes sufficiently large, $\Psi(Y)$ diminishes to zero in the middle of the channel. This is due to the important assumptions of Zeta potential [6] in the application of micro-channels (i) the surface is flat and (ii) the double layer is able to develop fully so that the potential in the middle of the channels is zero. It is also noticed that the HAM solutions agree with the analytical ones (10) in the whole region $-1 \leq Y \leq 1$ for all considered $\kappa$. 
The solution of Mala et al. (58) for the electrostatic potential exists singularity at $Y=0$, causes the velocity profiles discontinuous at the same place, the less is $\kappa$, the clearer is the discontinuity, as shown in Fig.3. Obviously, the solution is against the natural laws and physically impossible, while the present analytical solution (30) is consecutive and smooth in the whole region. Particularly when $\kappa$ is sufficiently large, Mala's result seems to match the present solution, but in this case, the distance between two plates is too large to describe the microchannel flow with interfacial electrokinetic effects. In Fig.3, it is seen that the HAM approximation agrees well with the analytical solution (30). This further confirms the validity and accuracy of the present HAM solutions.

The influence of $\kappa$ on the pressure constant $G_{1}$ is presented in Fig.4. It is shown that $G_{1}$ decreases monotonously as $\kappa$ evolves. It is noted that as the value of $\kappa$ decreases, the microchannel is narrower, thus the electric double layer effect is stronger. The electric double layer leads to back flow near the channel wall which could induce a stronger flow resistance in the microchannel, thus further lead the pressure constant increases. On the contrary, as $\kappa$ increases, the distance between the microchannel wall becomes larger, the electric double layer effect is weak, the fluid flow is not affected by electric double layer, so the pressure tends to be a constant. Viewed from another perspective, if we keep the distance between the two plates remains unchanged, then $\kappa$ is inversely proportional to the thickness of electrical double layer. The larger is $\kappa$, the thinner is the thickness of electric double layer. As $\kappa$ is considerably large, the thickness of electric double layer approaches to zero. In this situation, the electrical double layer effect on the fluid motion can also be ignored.

The streaming potential $E_{s}$ is an important factor to affect the flow patterns in the microchannel. As shown in Fig. $5, \bar{E}_{s}$ decreases very quickly as $\kappa$ evolves. It is sufficient to 
show that $\bar{E}_{s}$ approaches to zero with $\kappa$ being considerably large. In fact, the reason of this phenomenon can be explained from the point of the electrical double layer. The rising value of $\kappa$ may be due to the increasing bulk concentration of ions. Accordingly, the counter-ions concentration in the liquid increases, which leads to the diffuse layer compressed. With more counter-ions being extruded into the shear plane, the zeta potential is thus decreased leading to a decreased streaming potential. If the bulk concentration is sufficiently large, namely the $\kappa$ is large enough, the zeta potential will tend to zero, therefore the streaming potential also tend to zero.

In macroscopical flows, the effect of viscous dissipation on the fluid flow is usually small, therefore it could be ignored. But in microscopic ones, the viscous dissipation function can be very strong, and in turn its influence on the distribution of the flow temperature can be significant. As a result, it leads to the flow obviously changed in the microchannel. As seen in Fig.6, $\Theta(Y)$ increases rapidly with $E c$ increasing. It is also shown in Fig.7 that $E c$ has a significant effect on the nanoparticle concentration distribution $\Phi(Y)$. Its increase causes the rapid reduction of the relative nanoparticle concentration $\Phi(Y)$.

It is noted that the physical quantities such as the local skin friction, the local Nusselt number and the local Sherwood number are of importance in practical applications. It is therefore necessary to further examine discuss their variational trends with $R e$ and $\kappa$ on the lower wall in detail.

Many studies on microchannel flow, especially contains the effect of electric double layer, but less research with focus on Reynolds number. Thus, some results about the Reynolds number are presented in order to provide theoretical basis for test. From Table 3, it is found that the Reynolds number $R e$ have great influence on both the local Nusselt number $\mathrm{Nu}_{2}$ and the local Sherwood number $S h_{2}$. The absolute value of $N u_{2}$ decreases with the 
increase of $R e$. However, the value of $S h_{2}$ enhances with $R e$ evolving. It is also noted that $S h_{2}$ increases faster than $N u_{2}$ indicating that the effect of $R e$ on $S h_{2}$ is greater than that of $N u_{2}$. As shown in Fig.8, it is seen that $C_{f 2}$ decreases rapidly as $\kappa$ increases when $\kappa$ is small. It is worth mentioning that $C_{f 2}$ reaches a minimum near $\kappa=1.5$. Once going over this minimum value, $C_{f 2}$ increases as $\kappa$ evolves and approaches to 3 as $\kappa$ keeps continuously increasing. It is observed that the large $\kappa$ corresponds to the thin electric double layer thickness. Conversely, the small $\kappa$ indicates the thick electric double layer thickness. It is illustrated in Fig.9 that the variation of local Nusselt number $N u_{2}$ with $\kappa$ exhibits the totally reverse trend relative to that of $C_{f 2}$. The local Nusselt number $N u_{2}$ rapidly increases to its maximum value, then gradually reduces as $\kappa$ continuously increases. When $\kappa$ becomes sufficiently large, the local Nusselt number $N u_{2}$ closes to a certain constant. As shown in Fig.10, the influences of $\kappa$ on the local Sherwood number $S h_{2}$ are presented. It has the similar trend as the variation of $C_{f 2}$ with $\kappa$. Namely, $S h_{2}$ decreases as $\kappa$ increases for small $\kappa$. After reaching the minimum value, $S h_{2}$ increases as $\kappa$ evolves.

\section{Conclusion}

The nanofluid flow through a microchannel with the effects of the EDL are investigated. By eliminating the unphysical assumption leading to the discontinuities of flow field and replacing the inappropriate pressure constant assumption, the microchannel problem is reformulated, which is consistent with commonly-accepted models in channels. The major findings contained in this paper are as follows:

1. The analytical solutions for both the electrostatic potential and the velocity field are given, which are consecutive and smooth in the whole region. These results are obviously better than those given by Mala et al. [8]. 
2. $\kappa$ is a key factor to measure the EDL effects. It is noted that when $\kappa \geq 20$, its effects become negatively small. In this situation, the electrical double layer effect on the fluid motion and heat transfer can be ignored, thus the corresponding physical quantities tend to be constant, i.e. the streaming potential $\bar{E}_{s}$ approaches to zero. The pressure constant $G_{1}$, the local skin friction, the local Nusselt number and the local Sherwood number approach to fixed values, respectively.

3. The effect of viscous dissipation influence on the distribution of the fluid temperature and the nanoparticle concentration is significant. The reason is that the shear strength and frictional resistance are greatly increased in the microchannel, it leads to the viscous dissipation function is very strong.

\section{References}

[1] M. R. Safaei, M. Gooarzi, O.A. Akbari, M.S. Shadloo, M. Dahari, Performance evaluation of nanofluids in an inclined ribbed microchannel for electronic cooling applications. Electronics Cooling, (2016), DOI: 10.5772/62898.

[2] A. A. A. Arani, O. A. Akbari, M. R. Safaei, A. Marzban, A.A.A.A. Alrashed, Heat transfer improvement of water/single-wall carbon nanotubes (SWCNT) nanofluid in a novel design of a truncated double-layered microchannel heat sink. International Journal of Heat and Mass Transfer, 113, (2017) 780-795.

[3] A. B. Duncan, G. P. Peterson, Review of microscale heat transfer. Applied Mechanics Reviews, 47(9), (1994) 397-428.

[4] X. F. Peng, G. P. Peterson, B. X. Wang, Heat transfer characteristics of water flowing through microchannels. Experimental Heat Transfer, 7(4), (2012) 265-283. 
[5] B. X. Wang, X. F. Peng, Experimental investigation on liquid forced-convection heat transfer through microchannels. International Journal of Heat and Mass Transfer, $37,(1994) 73-82$.

[6] R.J. Hunter, Zeta Potential in Colloid Science, Principles and Applications. Academic Press, New York, 1981.

[7] C. Yang, D. Li , Analysis of electrokinetic effects on the liquid flow in rectangular microchannels. Colloids and Surfaces A: Physicochemical and Engineering Aspects, 143(2),(1998) 339-353.

[8] G. M. Mala, D. Li, J. D. Dale, Heat transfer and fluid flow in microchannels. International Journal of Heat and Mass Transfer, 40(13),(1997) 3079-3088.

[9] G. M. Mala, D. Li, C. Werner, H. J. Jacobasch, Y. B. Ning, Flow characteristics of water through a microchannel between two parallel plates with electrokinetic effects. International Journal of Heat and Fluid Flow, 18(5),(1997) 489-496.

[10] L. Ren, W. Qu, D. Li,Interfacial electrokinetic effects on liquid flow in microchannels. International Journal of Heat and Mass Transfer, 44(16),(2001) 3125-3134.

[11] Y. P. Zhang, T. W. Xu, Z. H. Liu, Streaming potential across a porous charged membrane in organic-aqueous solutions. Desalination, 212(1-3), (2007) 183-190.

[12] M. R. Safaei, A. H. Jahanbin, A. Kianifar, S. Gharehkhani, A.S. Kherbeet, Mathematical modeling for nanofluids simulation: a review of the latest works. Modeling and Simulation in Engineering Sciences, (2016), DOI: 10.5772/64154.

[13] A. Karimipour, A. H. Nezhad, A. D’Orazio, M. H. Esfe, M. R. Safaei, Simulation of copper-water nanofluid in a microchannel in slip flow regime using the lattice Boltzmann method. European Journal of Mechanics - B/Fluids, 49(49), (2015) 89-99. 
[14] Z. Nikkhah, A. Karimipour, M. R. Safaei, P. Forghani-Tehrani, M. Goodarzi , Forced convective heat transfer of water/functionalized multi-walled carbon nanotube nanofluids in a microchannel with oscillating heat flux and slip boundary condition. International Communications in Heat and Mass Transfer, 68, (2015)69-77.

[15] D. Wen, Y. Ding, Experimental investigation into convective heat transfer of nanofluids at the entrance region under laminar flow conditions. International Journal of Heat and Mass Transfer, 47(24),(2004) 5181-5188.

[16] W. Williams, J. Buongiorno, L. W. Hu, Experimental investigation of turbulent convective heat transfer and pressure loss of alumina/water and zirconia/water nanoparticle colloids (nanofluids) in horizontal tubes. Journal of Heat Transfer, 130(4), (2008) 042412

[17] M. R. Safaei, M. S. Shadloo, M. S. Goodarzi, A. Hadjadj, H. R. Goshayeshi , A survey on experimental and numerical studies of convection heat transfer of nanofluids inside closed conduits. Advances in Mechanical Engineering,8(10),(2016).

[18] Y. Xuan, Q. Li, Investigation on convective heat transfer and flow features of nanofluids. Journal of Heat transfer, 125(1),(2003) 151-155.

[19] Y. Yang, Z. G. Zhang, E. A. Grulke, W. B. Anderson, G. Wu, Heat transfer properties of nanoparticle-in-fluid dispersions (nanofluids) in laminar flow. International Journal of Heat and Mass Transfer, 48(6),(2005) 1107-1116.

[20] S. U. S. Choi, Enhancing thermal conductivity of fluids with nanoparticles, in: Proceedings of the 1995 ASME International Mechanical Engineering Congress and Exposition FED 231/ MD66, ASME, San Francisco, CA, USA, (1995) 99-106.

[21] Y. Xuan, W. Roetzel, Conceptions for heat transfer correlation of nanofluids. International Journal of Heat and Mass Transfer, 43(19),(2000) 3701-3707. 
[22] J. Buongiorno, Convective transport in nanofluids. Journal of Heat Transfer ASME, 128(3), 2006 240-250.

[23] O. A. Bég, M. F. M. Basir, M. J. Uddin, A. I. M. Ismail, Numerical study of slip effects on unsteady asymmetric bioconvective nanofluid flow in a porous microchannel with an expanding/contracting upper wall using buongiornos model. Journal of Mechanics in Medicine and Biology, (2016)1750059.

[24] S. Tardu, The electric double layer effect on the microchannel flow stability. Microscale Thermophysical Engineering, 8(4),(2004) 383-401.

[25] A. S. Lavine, Analysis of fully developed opposing mixed convection between inclined parallel plates. Wärme - und Stoffübertragung, 23(4),(1988) 249-257.

[26] X. C. You, H. Xu, I. Pop, Analysis of fully developed opposing mixed convection flow in an inclined channel filled by a nanofluid, Journal of Heat Transfer ASME, 136,(2014) 124502-1-124502-5,

[27] S. J. Liao Homotopy analysis method in nonlinear differential equations. Higher Education Press; Springer, 2012. 
Table 2: The maximum error $E(m)$

\begin{tabular}{ccccc}
\hline order & $\kappa=1$ & $\kappa=3$ & $\kappa=5$ & $\kappa=20$ \\
\hline 10 & 1711.196 & 13738.398 & 4686.691 & 70.382 \\
20 & 8.002 & 0.793 & 0.125 & 0.798 \\
30 & $1.87 \times 10^{-7}$ & 0.166 & $2.80 \times 10^{-4}$ & $6.50 \times 10^{-4}$ \\
40 & $5.37 \times 10^{-15}$ & $1.49 \times 10^{-4}$ & $1.42 \times 10^{-8}$ & $2.82 \times 10^{-5}$ \\
50 & $1.32 \times 10^{-23}$ & $7.17 \times 10^{-7}$ & $1.24 \times 10^{-8}$ & $1.097 \times 10^{-5}$ \\
60 & - & - & - & $1.46 \times 10^{-6}$ \\
\hline
\end{tabular}


Table 3: The results of $N u_{2}$ and $S h_{2}$ with variation of $R e$ and $\kappa$ in the case of

\begin{tabular}{|c|c|c|c|c|c|c|}
\hline \multirow{2}{*}{$\operatorname{Re}$} & \multicolumn{2}{|c|}{$\kappa=1$} & \multicolumn{2}{|c|}{$\kappa=3$} & \multicolumn{2}{|c|}{$\kappa=20$} \\
\hline & $N u_{2}$ & $S h_{2}$ & $N u_{2}$ & $S h_{2}$ & $N u_{2}$ & $S h_{2}$ \\
\hline 1 & -9.090 & 9.545 & -9.119 & 9.559 & -9.180 & 9.590 \\
\hline 10 & -2.088 & 51.044 & -2.137 & 51.069 & -2.247 & 51.123 \\
\hline 20 & -1.216 & 100.608 & -1.249 & 100.625 & -1.328 & 100.664 \\
\hline 30 & -0.843 & 150.421 & -0.867 & 150.433 & -0.927 & 150.464 \\
\hline 40 & -0.643 & 200.326 & -0.662 & 200.331 & -0.711 & 200.356 \\
\hline 50 & -0.520 & 250.291 & -0.536 & 250.269 & -0.576 & 250.291 \\
\hline
\end{tabular}




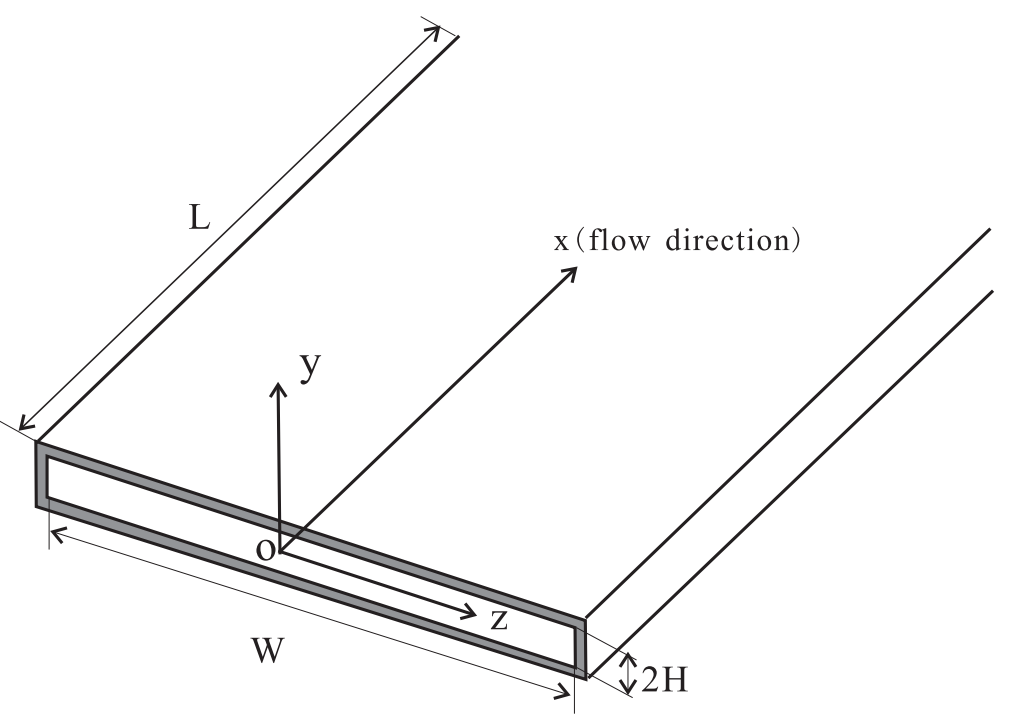

(a) 3-D sketch

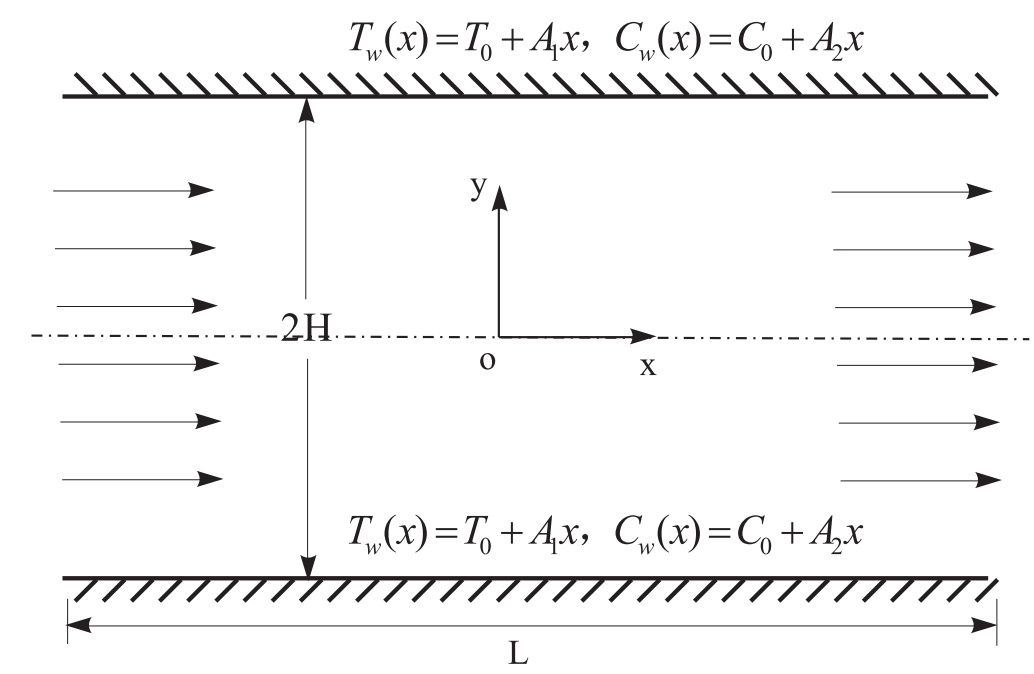

(b) 2-D sketch

Figure 1: Physical model and coordinate system 


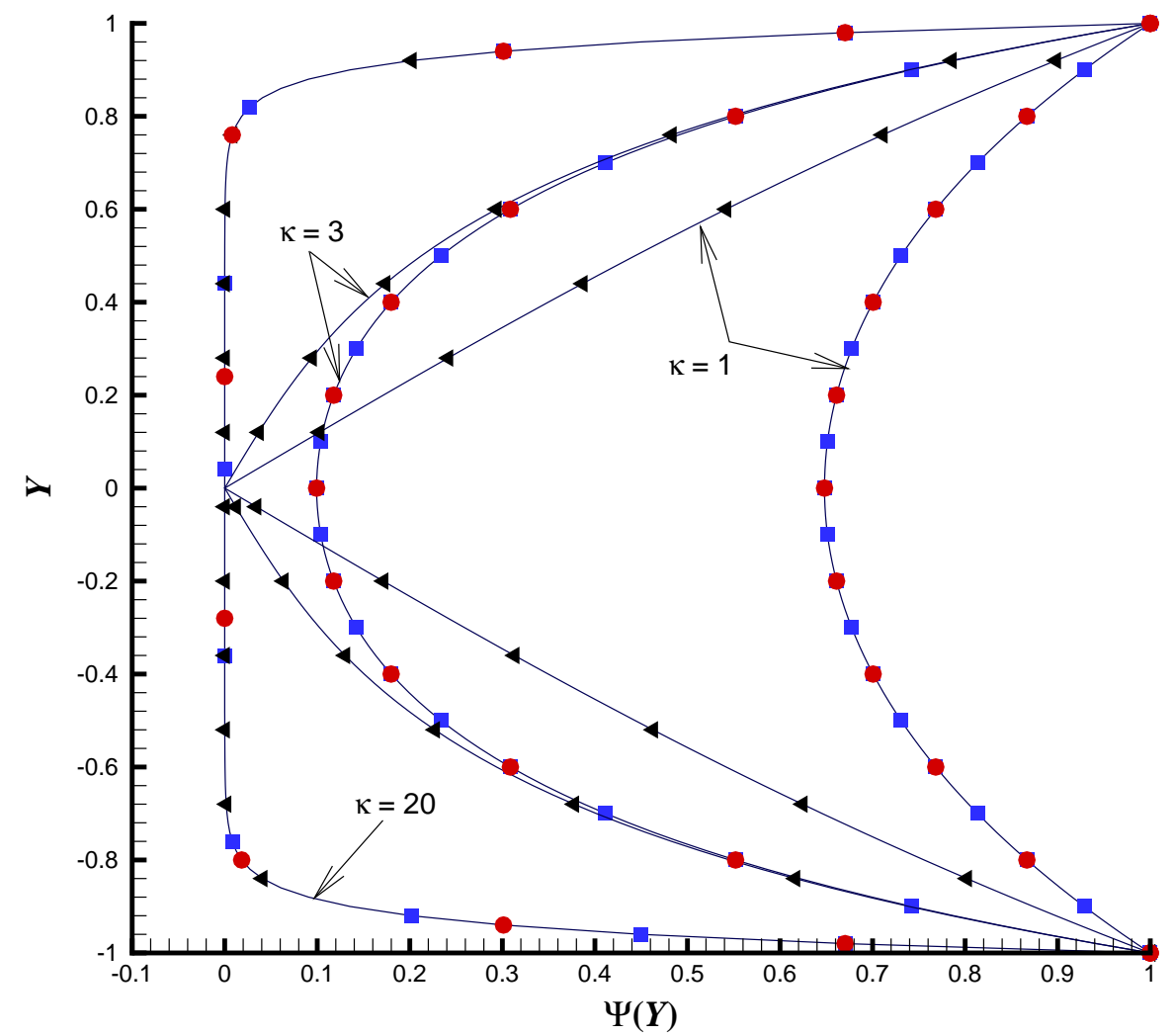

Figure 2: The electrical potential $\Psi(Y)$ for various values of $\kappa$ with $\bar{\zeta}=1$. Line with circles: analytical solutions given by Eq.(10); line with squares: HAM solutions; line with gradients: results of Mala et al. [8]. 


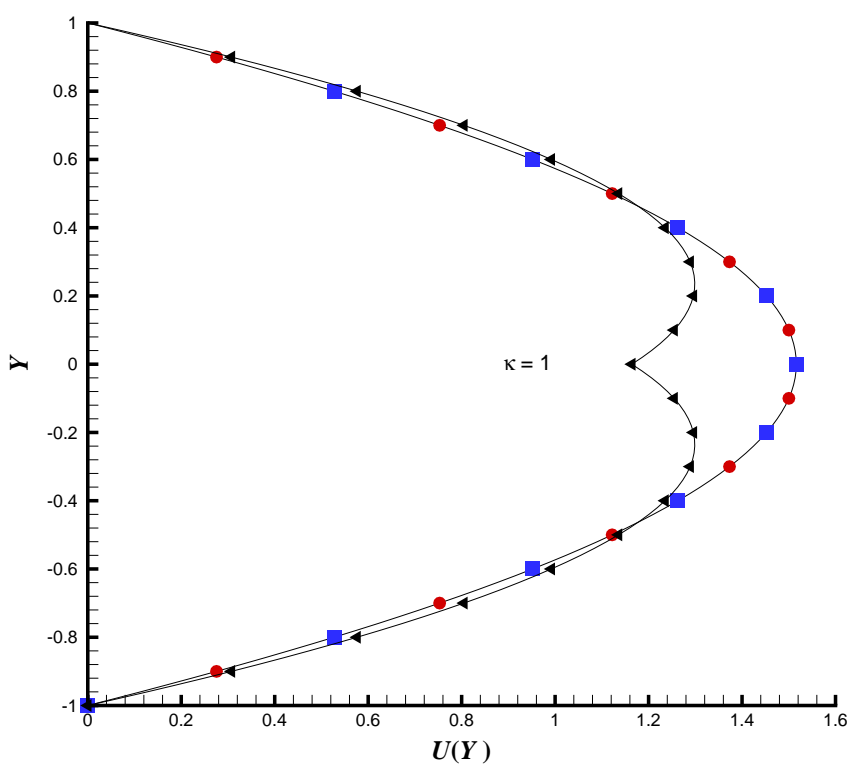

(a) $\kappa=1$

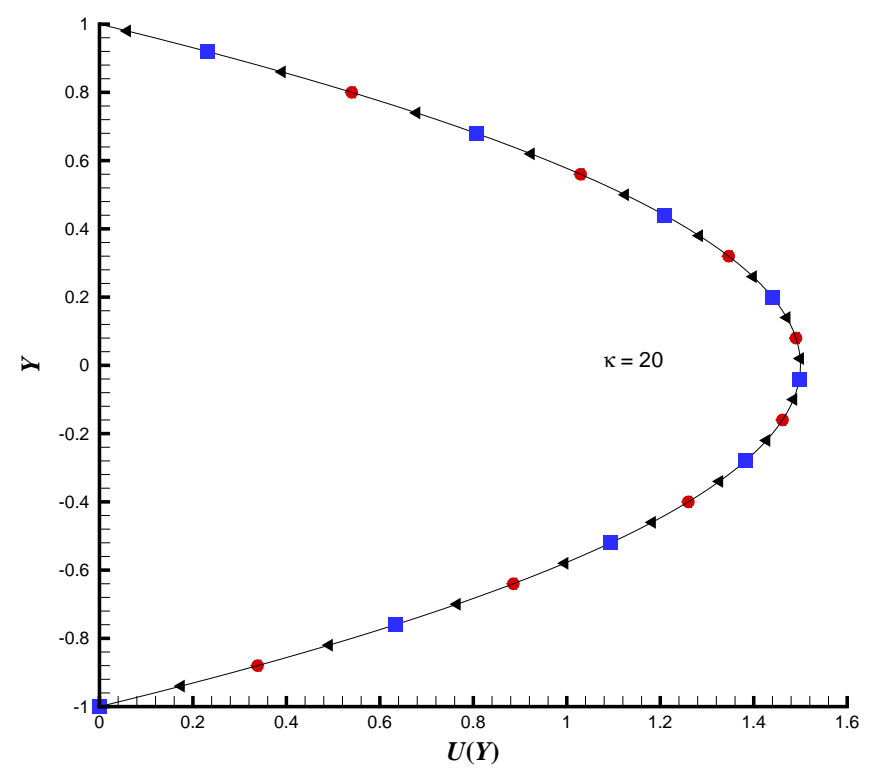

(b) $\kappa=20$

Figure 3: The velocity $U(Y)$ for various values of $\kappa$ with $G_{2}=G_{3}=1$ and $\bar{\zeta}=1$. Line with circles: analytical solutions given by Eq.(10); line with squares: HAM solutions; line with gradients: results of Mala et al. [8]. 


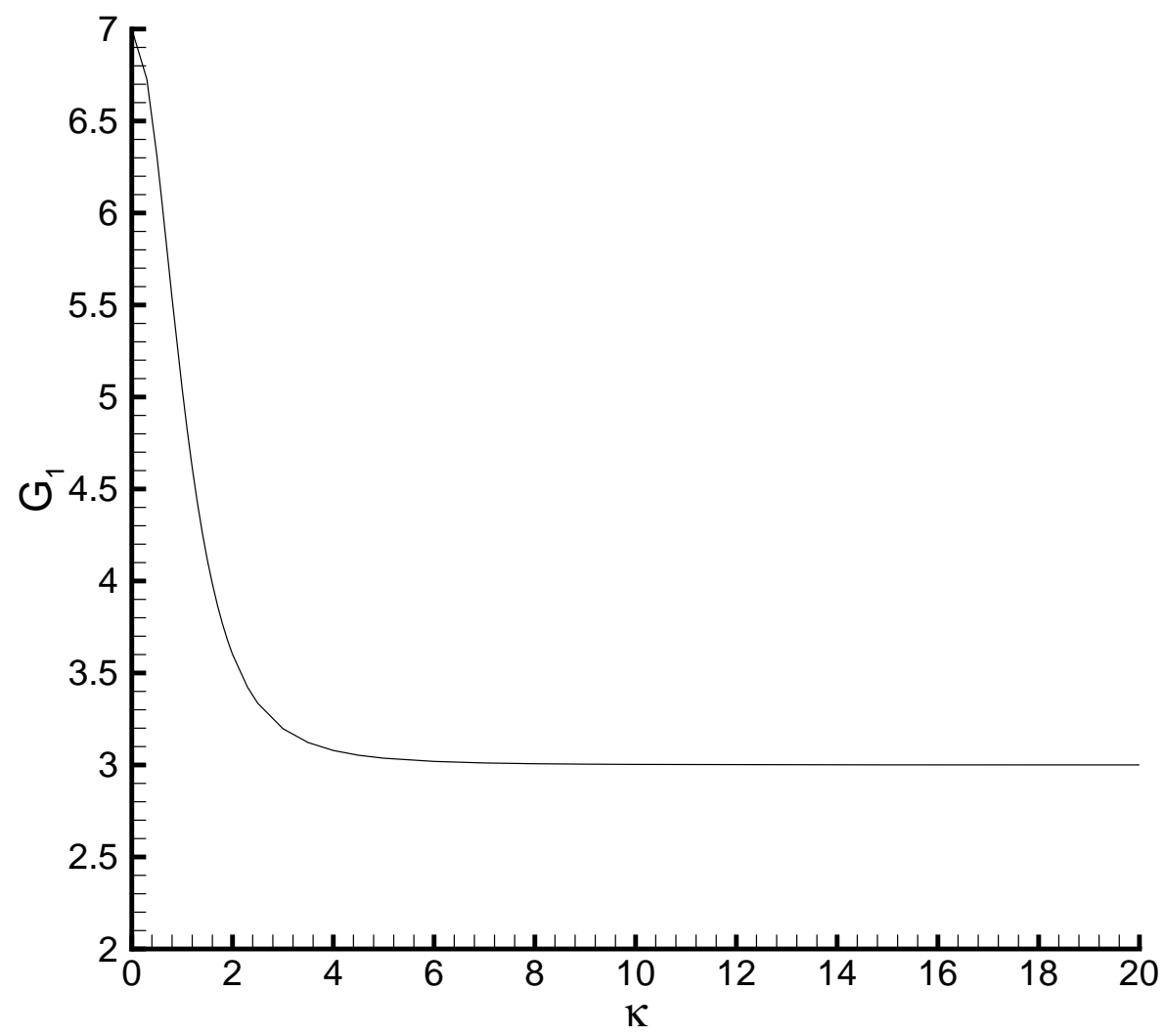

Figure 4: Variation of the pressure constant $G_{1}$ with $\kappa$ in the case of $E c=L e=$ $R e=1, N b=1 / 5, N t=1 / 10, \operatorname{Pr}=5, G_{2}=G_{3}=1$ and $\bar{\zeta}=1$. 


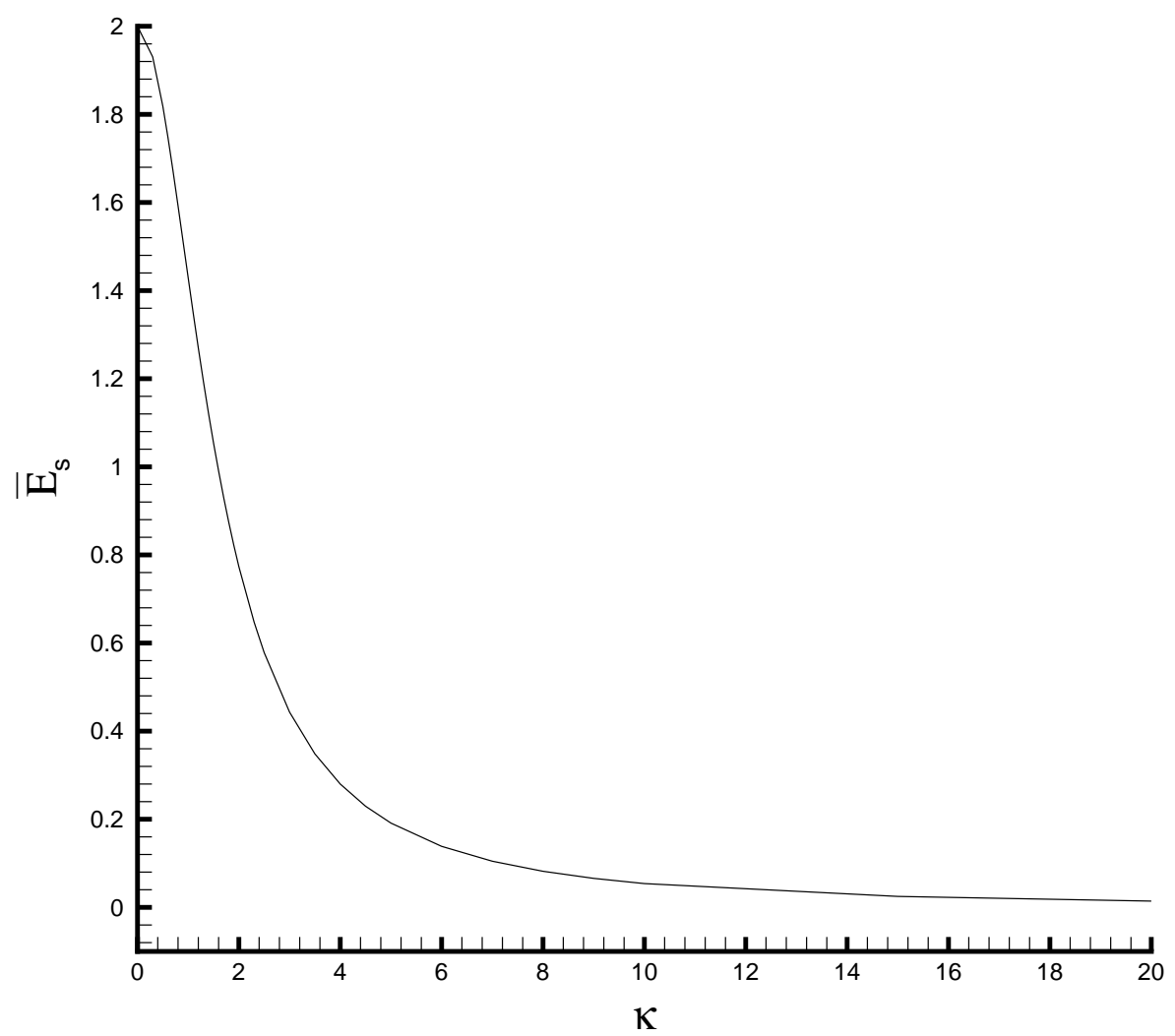

Figure 5: Variation of the streaming potential $\bar{E}_{s}$ with $\kappa$ in the case of $E c=L e=$ $R e=1, N b=1 / 5, N t=1 / 10, \operatorname{Pr}=5, G_{2}=G_{3}=1$ and $\bar{\zeta}=1$. 


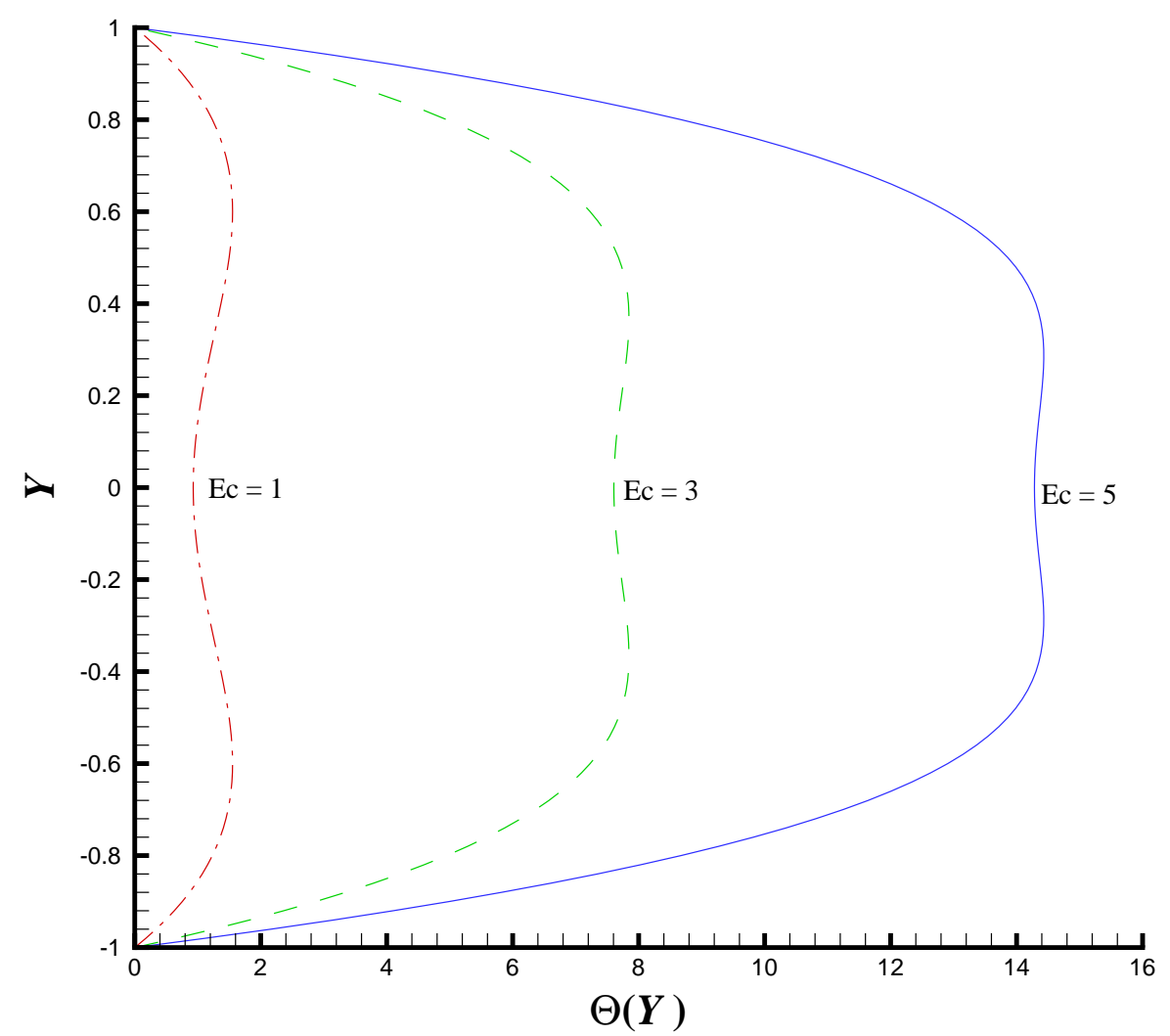

Figure 6: Variation of $\Theta(Y)$ with $E c$ in the case of $\kappa=1, L e=R e=1, N b=1 / 5$, $N t=1 / 10, \operatorname{Pr}=5, G_{2}=G_{3}=1$ and $\bar{\zeta}=1$. 


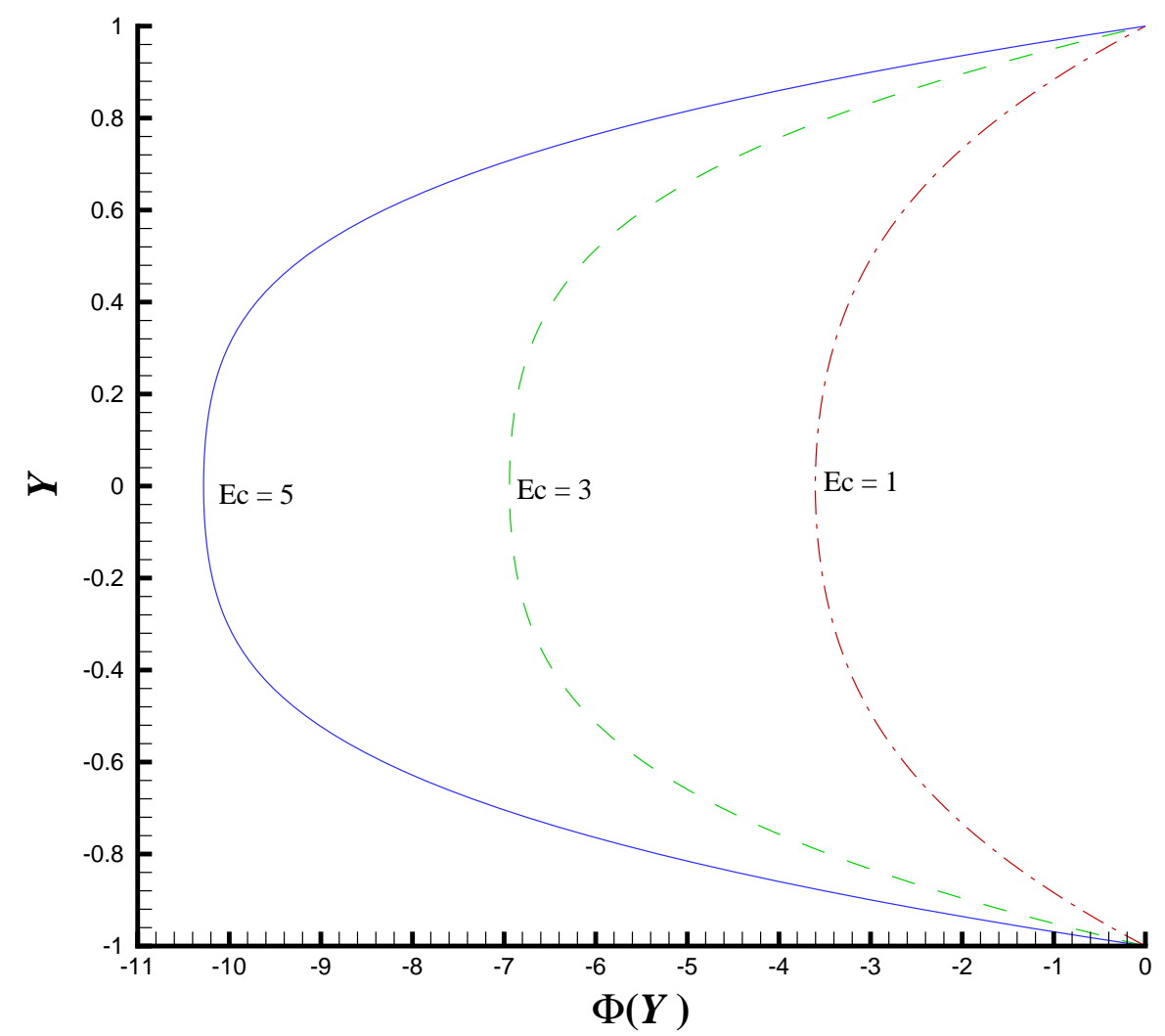

Figure 7: Variation of $\Phi(Y)$ with $E c$ in the case of $\kappa=1, L e=R e=1, N b=1 / 5$, $N t=1 / 10, \operatorname{Pr}=5, G_{2}=G_{3}=1$ and $\bar{\zeta}=1$. 


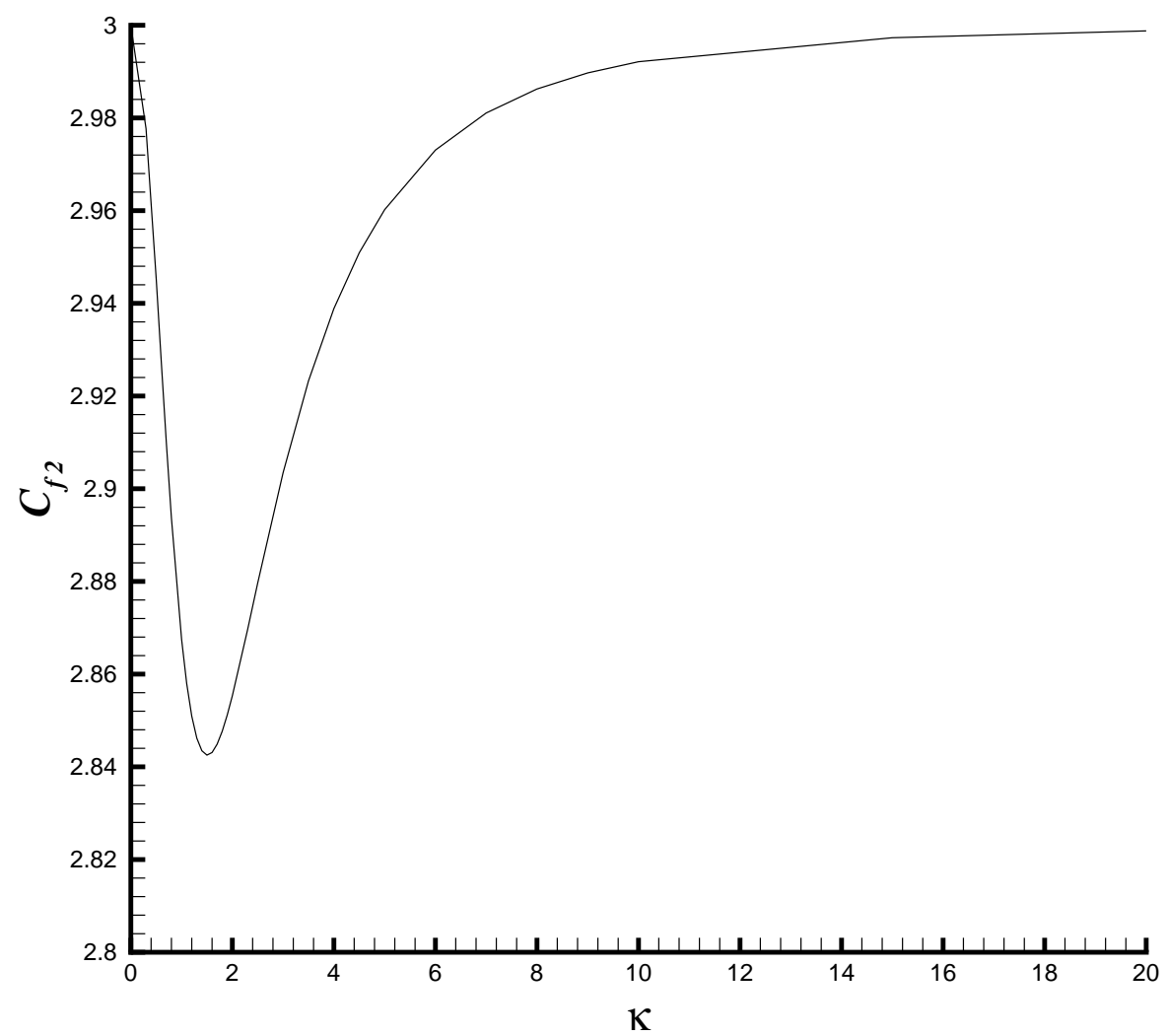

Figure 8: Variation of $C_{f 2}$ with $\kappa$ in the case of $E c=L e=R e=1, N b=1 / 5$, $N t=1 / 10, \operatorname{Pr}=5, G_{2}=G_{3}=1$ and $\bar{\zeta}=1$. 


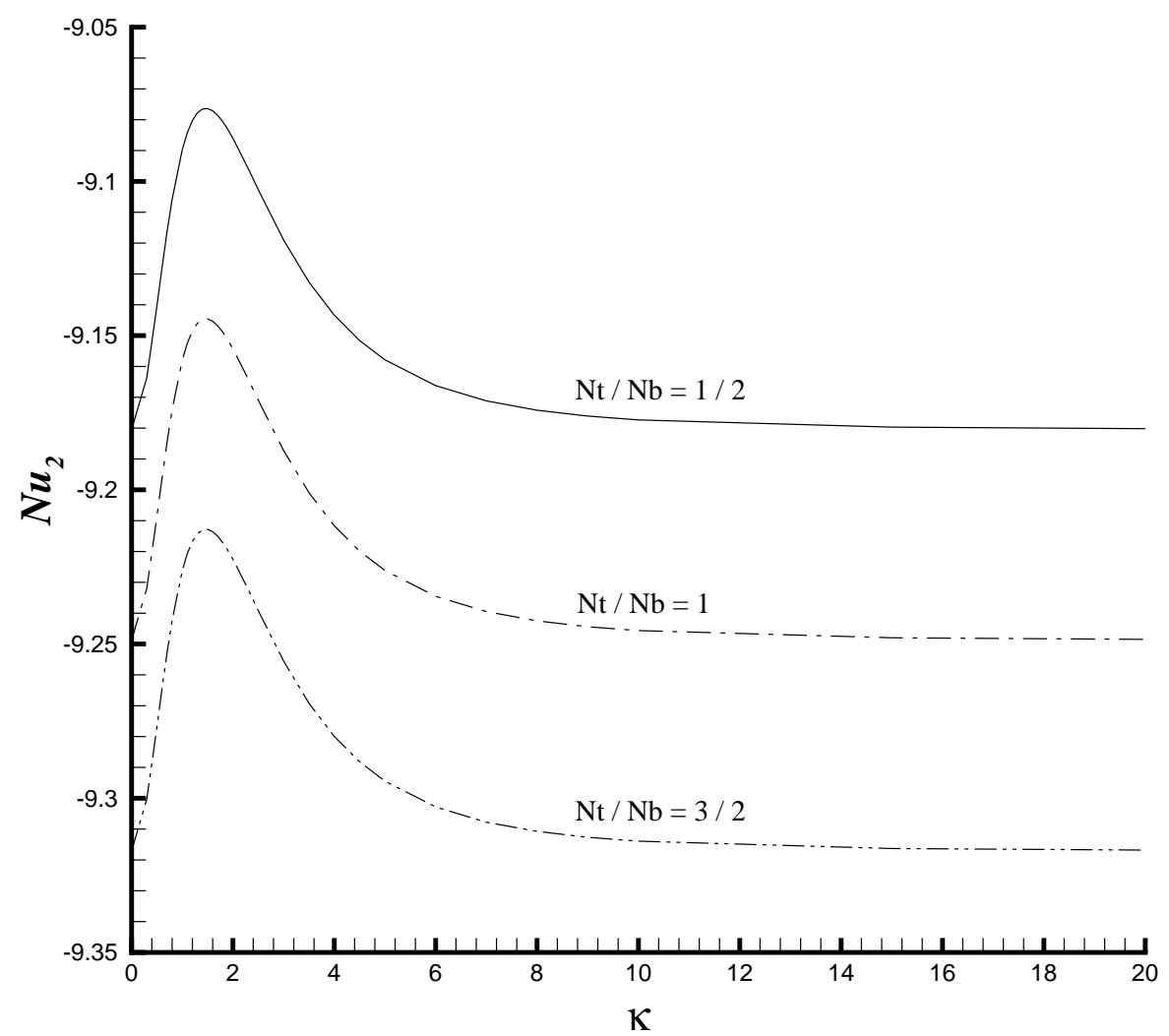

Figure 9: Variation of $N u_{2}$ with $\kappa$ in the case of $E c=L e=R e=1, \operatorname{Pr}=5$, $G_{2}=G_{3}=1$ and $\bar{\zeta}=1$. 

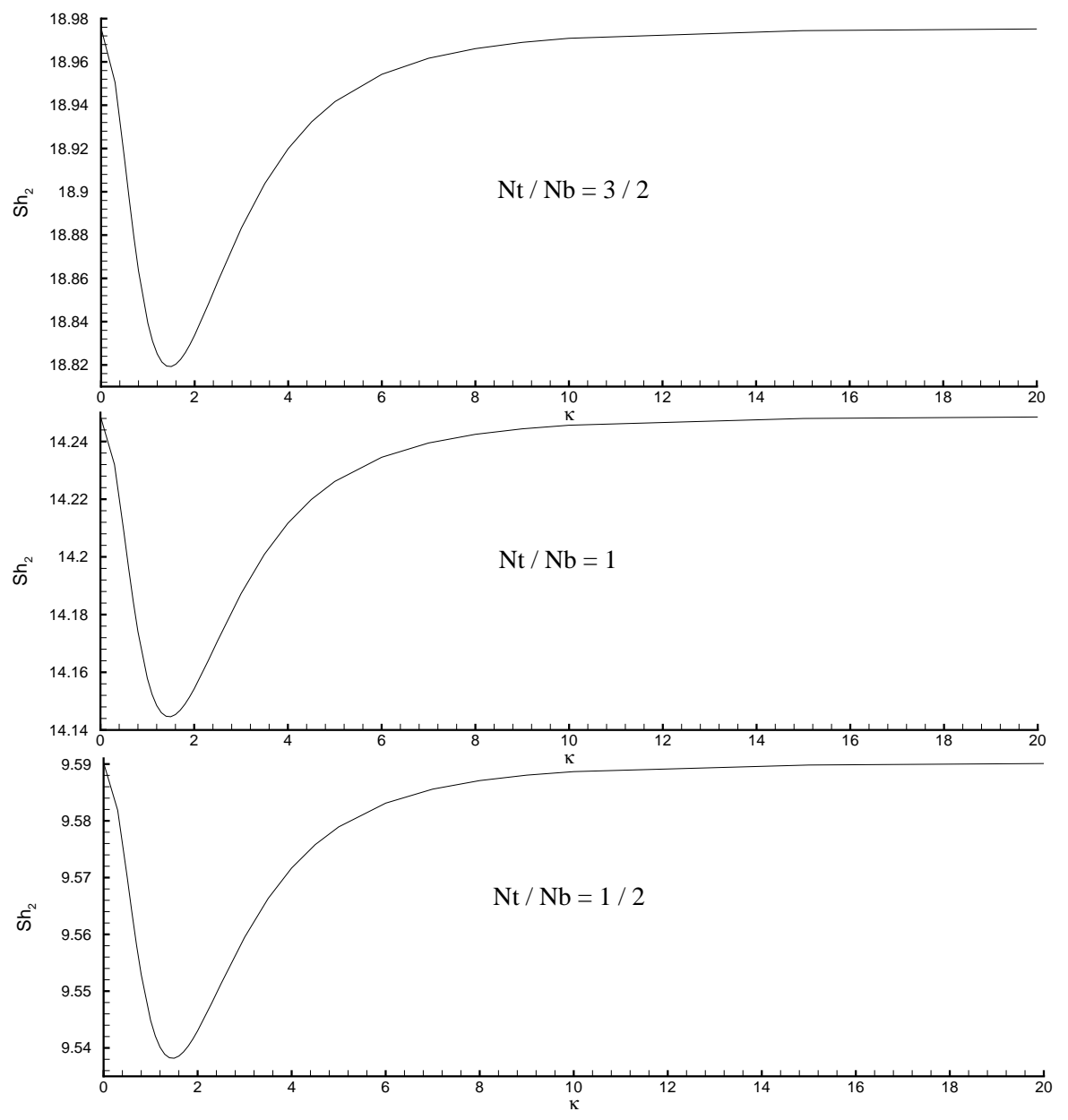

Figure 10: Variation of $S h_{2}$ with $\kappa$ in the case of $E c=L e=\operatorname{Re}=1, \operatorname{Pr}=5$, $G_{2}=G_{3}=1$ and $\bar{\zeta}=1$. 